\title{
In-Bus: Diseño de información para rutas de autobús en la zona metropolitana de Guadalajara
}

\author{
Sarah Ventura, Zuli Galindo, Juliana Avila, Carlos \\ Díaz de León, Carlos Aceves
}

Publicado: 21 Septiembre 2016

\begin{abstract}
Resumen
Las actividades instrumentales de la vida diaria se contemplan como un aspecto necesario para tener un desempeño independiente en la comunidad. Una de las actividades instrumentales significativamente correlacionada con el estado de salud es el uso de transporte público (Seidel, 2009). Bajo el enfoque de Ergonomía Participativa, el objetivo de esta propuesta de diseño es mejorar la usabilidad de los mapas de las rutas de camión mediante la participación de adultos mayores durante el proceso de rediseño de dicha interface (mapa). En el estudio se incluyeron 77 adultos mayores de la Zona Metropolitana de Guadalajara (ZMG), pertenecientes a diferentes colonias de la ciudad y en su mayoría, usuarios asiduos del Sistema de Transporte Público (STP). La evaluación del mapa se realizó con el Post-Study System Usability Questionnaire (PSSUQ), instrumento que mide la satisfacción del usuario después de la interacción con la interfaz, basada en una tarea. Como resultado del análisis se establecieron lineamientos de diseño que condujeron al rediseño del mapa actual.
\end{abstract}

Palabras clave: Interacción Humano-Computadora; Diseño participativo; Transporte; Adultos mayores.

\section{Introducción}

En la vida cotidiana, el trabajo del planificador urbano y del diseñador es el que permite navegar por las ciudades de manera eficiente, con el fin de que sus habitantes puedan transitar en ella, de acuerdo con sus capacidades; es decir, aún para aquellas personas consideradas como vulnerables. Para lograrlo, se hace necesario tener en cuenta el concepto de usabilidad propuesto por Nielsen, quien la define como un atributo o cualidad que evalúa qué tan fácil de usar son las interfaces; la palabra "usabilidad" también se refiere a los métodos utilizados durante el proceso de diseño para mejorar la facilidad de uso [1]. Con ello, desde la perspectiva de la Ergonomía, se facilita a las personas la comprensión e interacción con las interfaces gráficas, cometiendo

Ventura, S., Galindo, Z., Avila, J., Díaz de León, C., Aceves, C. Universidad de Guadalajara

Calzada Independencia Norte 5075

C.P.44250, Guadalajara, Jalisco.

Email: saritahven@gmail.com, zulig89@gmail.com, juliana.avila.vargas@gmail.com, dilezul@gmail.com, c.aceves@academicos.udg.mx el menor número de errores.

\section{Planteamiento del problema}

El envejecimiento poblacional es un fenómeno demográfico señalado por las Naciones Unidas [2] como el proceso de rápido aumento en el número de personas mayores (+60 años) en la población total. Este fenómeno es considerado como uno de los grandes retos mundiales debido a la demanda social y económica que implica [3]. La Organización Mundial de la Salud [4] propone difundir el proceso de envejecimiento activo para optimizar las oportunidades de salud, participación y seguridad con el fin de mejorar la calidad de vida durante esta etapa. Dicho proceso de envejecimiento activo se relaciona con las actividades instrumentales de la vida diaria, necesarias para una vida independiente en la comunidad. Una de las actividades instrumentales significativamente correlacionada con el estado de salud es el uso de transporte público [5]. En el caso particular de los adultos mayores, el acceso al transporte público puede ayudarlos a proveerse de bienes, servicios, empleo y otras actividades, que le permiten mejorar su sentido de libertad e independencia [6]. Las Naciones Unidas [7] ha puntualizado la necesidad de generar y adaptar ciudades con accesibilidad universal, para ello ha planteado lineamientos básicos con los que debe contar el equipamiento urbano, señalética y el diseño de los espacios de desplazamiento peatonal.

Un estudio sobre el uso del transporte público en la ciudad de Guadalajara determinó cuáles son los mayores problemas que enfrenta la población al usar éste servicio, con el fin de mejorarlo y hacerlo inclusivo, lo cual se realizó mediante grupos de enfoque con adultos mayores y jóvenes. Los primeros reportaron la carencia de información sobre las rutas y los cambios en las mismas, siendo esto uno de los nueve problemas más significativos [8]. Sumado a esto, se ha encontrado que las personas mayores necesitan más información previa a su viaje, a causa de la disminución de sus capacidades físicas y mentales [9].

El diseño de la información consiste en la preparación de la misma, de manera que puede ser usada por las personas con eficiencia y eficacia, con lo cual se busca resolver muchos problemas durante el diseño de interfaces humano-computadora [10]. El tipo y el grado de interacción que tiene el usuario con una interfaz es uno de los factores que se debe considerar en el diseño de las mismas [11]. Los mapas de rutas de transporte cuentan con un output más no un input, lo que significa que el usuario no podrá ingresar datos o manipular elementos de las 
distintas interfaces pero sí podrá recibir información de ellas (output).

En relación con lo anterior, debe ser considerado el concepto de usabilidad durante el diseño de interfaces para que sean más fáciles de usar y aprender [12], haciendo que estas reflejen convenios del mundo real, estándares, orden y minimicen la carga mental al ejecutar las tareas [13]. El incremento de la usabilidad en los sistemas de información para pasajeros ha sido relacionada con el desarrollo de la calidad de los mismos [14]. De igual manera, una investigación en Estados Unidos, midió la usabilidad de la interfaz e información de un sistema de planeación de traslados en un sistema de transporte multimodal para encontrar aspectos relevantes a mejorar en el diseño de dicho sistema, además se reporta que los instrumentos utilizados permiten la evaluación de la reacción de los usuarios durante el uso de sistemas similares [15]. Por otra parte, un estudio reportó que la mejora del diseño y acceso a información del sistema de autobuses en Winchester elevó el número de usuarios y su satisfacción hacia el servicio [16]. Por tanto, el proceso de definición de requerimientos de usabilidad depende del claro entendimiento de los usuarios, tareas, contexto ambiental y de los sistemas [17].

No obstante, se debe abordar el concepto de Diseño Inclusivo, descrito como la comprensión de la diversidad de usuarios para mejorar las decisiones del proceso de desarrollo de productos y servicios, con el fin de satisfacer las necesidades de más personas [18]. Lo anterior es fundamental debido a que el porcentaje de adultos mayores va en aumento, esto como consecuencia del fenómeno de envejecimiento poblacional, latente en nuestra sociedad. A fin de brindar soluciones es inherente desarrollar diseños creativos e innovadores adaptados a sus necesidades, capacidades y expectativas, por medio de investigación y análisis desde el punto de vista ergonómico, contemplando las limitaciones motoras, sensoriales y cognitivas de la población de adultos mayores [19].

El objetivo de esta propuesta de diseño es mejorar la usabilidad de los mapas de las rutas de camión mediante la participación de los adultos mayores durante el proceso de diseño de información. El diseño de la información es una etapa de gran importancia para generar una solución más certera (precisa), tomando en consideración la adaptación del diseño propuesto a interfaces de computadora y dispositivos móviles con la finalidad de aumentar la accesibilidad a dicha información.

\section{Impacto social en el contexto mexicano}

En la ZMG viven alrededor de 4.5 millones de personas [20] las cuales tienen la necesidad de trasladarse por la ciudad diariamente. El $8.21 \%$ son la población de adultos mayores y se concentran en el municipio de Guadalajara y Zapopan. Según datos obtenidos del $28 \%$ al $35 \%$ de los viajes realizados en la ZMG se hacen a través del Sistema de Transporte Público (STP), 1 de cada 3 personas lo utilizan. Cabe resaltar que el resto de la población hace sus traslados a pie o en un automóvil particular, sin dejar de lado que este porcentaje es también un usuario potencial del STP. Julia Barret [21] menciona "Si los adultos mayores tienen la información que desean, en una forma que les sea fácil de entender y usar y proporcionada en métodos aceptables para ellos, serán más independientes y requerirán menos apoyo de los servicios de sociales y de salud". De igual manera el diseño resultante no solo será benéfico para la población de adultos mayores ya que también beneficiará a los demás usuarios del STP.

\section{Proceso de diseño}

\subsection{Lineamientos de diseño}

Se realizó una evaluación inicial de usabilidad al mapa que actualmente se encuentra a disposición del público dentro de los camiones, en la cual participaron adultos mayores de diferentes colonias de la ZMG. A partir de esta información se establecieron los lineamientos de diseño para el planteamiento de las nuevas propuestas. Los principales hallazgos se centraron en las características de la información que debía estar contenida en el mapa: ubicación de calles principales, colonias, hitos (puntos de referencia) y los lugares en los cuales se pueden realizar transbordos. Sobre las características del diseño se mencionó que debía tener un tamaño mayor y que el uso de color en el mapa sea el adecuado. Así mismo, se identificó que esta población prefiere encontrar la información de las rutas en los parabuses, dentro del camión o en folletos impresos.

\subsection{Diseño gráfico}

Se clasificó el trazado de las rutas, de acuerdo con el recorrido de las mismas: cerrado (loop) o con inicio y final diferente. Posteriormente se identificaron las calles principales según lo establecido en los Planes Parciales de Desarollo Urbano de los distintos municipios de la ZMG para señalarlas en el mapa, al igual que las colonias y los hitos cercanos a cada recorrido. Además de ello, se identificó en el sitio web de Rutas GDL (www.rutasgdl.com) las rutas que circulan por las calles principales que cruzan con el recorrido, esto con el fin de enlistarlas en cada mapa para facilitar al usuario la identificación de otras rutas que puedan ser de su interés o que pueda realizar un posible transbordo. Además, se establecieron los elementos gráficos como tipografía, tamaño de la misma y colores según las recomendaciones de Nielsen y Loranger [22] y Krung [23]. Finalmente, en el mapa se señalizaron hitos o lugares importantes, dirección, cruces y transbordos debido a que es considerada información fundamental cuando se transita en una zona desconocida [6] y son elementos clave para ayudar al usuario a navegar por la ciudad [24, 25].

\subsection{Configuración de la propuesta final}

Durante esta etapa se contó nuevamente con la participación adultos mayores quienes evaluaron la usabilidad de la propuesta realizada para mejorar la presentación de la información de una ruta de camión. Finalmente, la configuración de la propuesta resultante se ajustó con base en las recomendaciones dadas durante esta última evaluación, ver figura 1.

\section{Metodología}

La metodología del presente trabajo se basa en la Ergonomía participativa, enfoque para la implementación de tecnología que implica una alta participación de los usuarios finales en su desarrollo y aplicación [26]. La participación se denomina directa parcial, teniendo en cuenta que el grupo de usuarios seleccionados conforman sólo una parte de los grupos de interés.

\subsection{Participantes}

De acuerdo con esto, la prueba y encuestas se realizaron a 77 adultos mayores, 18 hombres y 59 mujeres, entre los 60 y 84 años de edad, con un promedio de 70 años. Todos los participantes son miembros del Centro Metropolitano del Adulto Mayor (CEMAM) de Guadalajara y 69 de ellos son usuarios frecuentes del STP. 


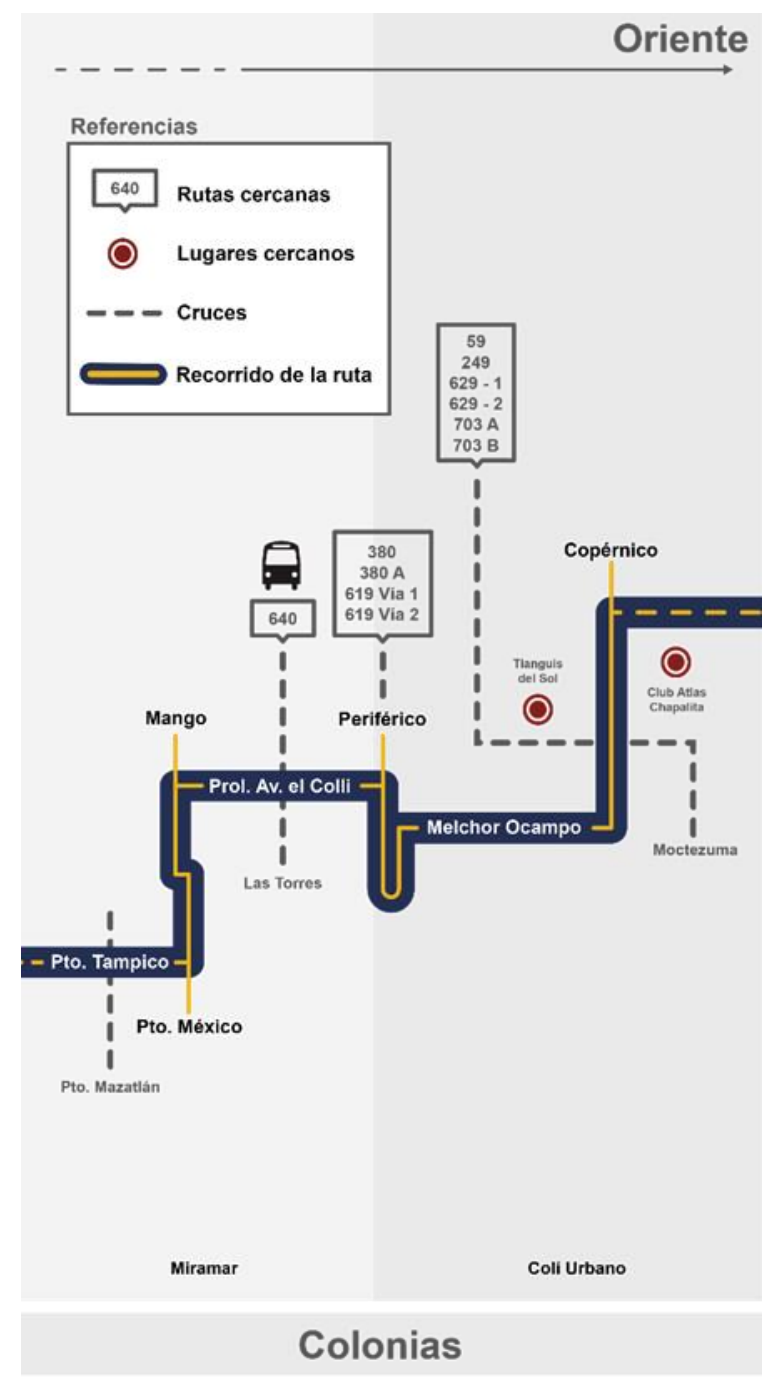

Figura 1. Sección de la propuesta final del mapa de la ruta 51

\subsection{Instrumentos}

Para la evaluación del mapa actual, se explicó a los participantes el procedimiento y se solicitó firmar el consentimiento escrito. Posteriormente, se les pidió señalar en el mapa en qué punto tomaran y otro donde descenderán de la ruta de acuerdo con las indicaciones del equipo de diseño. Se escogieron estos dos puntos ya que uno es una vía de tránsito principal sobre la cual pasa la ruta, y el segundo punto de referencia en un hito, la ruta pasa cerca de ahí. Se le indicó al participante que no había límite de tiempo y podía observar el mapa antes de hacer su selección.

Para realizar la evaluación del mapa se utilizó el Post-Study System Usability Questionnaire (PSSUQ) [27], instrumento que mide la satisfacción del usuario después de la interacción con la interfaz, basada en una tarea. Los ítems son calificados mediante escalas gráficas tipo Likert de siete puntos. Anclados en los extremos opuestos se encuentran las frases: "Totalmente de acuerdo" y "Totalmente en desacuerdo". La selección de los ítems se realizó en función de recomendaciones para la interacción y evaluación con población especial como adultos mayores y respetando el contenido integral con respecto a los constituyentes heurísticos de usabilidad. Los ítems seleccionados se dividen en subescalas, tres que evalúan la utilidad del sistema, uno la calidad de la interfaz, tres la calidad de la información y una última que completa el puntaje general de usabilidad. Se agregaron tres preguntas abiertas para recolectar información sobre: elementos a mejorar en el mapa, elementos que agregaría o eliminaría y para enlistar posibles ubicaciones de este tipo de información.

\section{Usuarios potenciales}

La información es una necesidad de todas las personas para poder comunicarse y desempeñar sus actividades diaria [10]. El diseño en general debe estar centrado en el reconocimiento de las capacidades de los usuarios, así como lo que demanda el producto para poder crear una compatibilidad en los mismos [28]. Durante el desarrollo de este proyecto se decidió contar con la participación de la población de adultos mayores como punto de referencia para el diseño de la información de las rutas de camión, puesto que al determinar las necesidades y las capacidades que debido al deterioro propio a la edad son más reducidas, lo cual incide en la interpretación y uso de la información. De manera que dentro de los usuarios potenciales se encuentran personas de cualquier edad que puedan leer y usen el transporte público, lo cual constituye el mayor porcentaje de la población que hace uso de dicho servicio.

\section{Solución propuesta}

\subsection{Organización de la información}

La información se organizó respetando la secuencia de la ruta, delimitando los giros que marca y su sentido (oriente - poniente). Se tomaron en cuenta lineamientos sugeridos que establecen que los espacios en blanco en una proporción 50/50 con la información hacen una interfaz más amigable y motiva a consultarla [29].

\subsection{Gráficos y simbología}

Los gráficos y la simbología funcionan mejor que las palabras para ayudar al usuario a identificar partes de la interfaz [29]. Estos elementos son importantes para identificar de manera rápida los ítems e información incluidos en el mapa y relaciones que se pueden generar entre las distintas rutas STP. Se hizo una distinción entre la figura y el fondo utilizando contorno de línea grueso de color negro o gris muy obscuro [30]. La simbología conserva homogeneidad y consistencia en toda la interfaz del mapa.

\subsection{Tipografía}

Se eligió la tipografía Arial recomendada en la literatura, con un rango tamaño en puntos de 12 hasta $\operatorname{los} 27$ (12, 14, 16 y 27 puntos) para generar jerarquía de la información a través del tamaño de letra. Esta tipografía nos proporciona buena legibilidad ya que no tiene adornos (sans serif), con la finalidad de abarcar un rango de edad más amplio y que los adultos mayores o personas con debilidad visual tuvieran mayores posibilidades para leerla. Se evitó el uso de texto solo en mayúsculas ya que la combinación de minúsculas con mayúsculas aumenta también la facilidad de lectura de los textos. Estas recomendaciones fueron consideradas según lo encontrado en la literatura [22, 23].

\subsection{Colores}

Para los textos se usó un contraste de letras negras o gris oscuro (90\%) con fondo gris claro $(10 \%)$, y blanco con fondo verde oscuro con la finalidad de mejorar contraste aumentando así la legibilidad y procurando llegar a los estándares del diseño universal combinado con la tipografía antes mencionada. Se utilizaron colores específicos para identificar el recorrido de la 
ruta (verde oscuro con naranja rojizo, alto contraste), gris oscuro (80\%) para los cruces con avenidas y transbordos con otras rutas. Se utilizaron como máximo cuatro colores en cada ilustración con base a las recomendaciones de Nielsen \& Loranger [22], sin tomar en cuenta el color blanco, el negro y su escala de grises intermedios dentro de esos cuatro.

\subsection{Tamaño}

El tamaño del mapa es de $80 \mathrm{~cm}$ por $32 \mathrm{~cm}$, un tamaño mayor al mapa original. Aun así en las últimas encuestas los participantes (17\%) sugieren de alguna manera que se aumente de tamaño, esto será posible ya que los participantes sugirieron que estuvieran en los parabuses (68\%) y los camiones (36\%), ambos cuentan con superficie suficiente para colocar el mapa hasta un ancho de $120 \mathrm{~cm}$.

\subsection{Eficacia y satisfacción}

En la tarea de ubicación de puntos referencia en el mapa actual se encontró que solo el $34 \%$ ejecutó la tarea correctamente, mientras que con la propuesta de diseño se obtuvo un mayor porcentaje de aciertos (84\%); con ello, la nueva propuesta parece generar una interacción más eficaz con los usuarios. De manera complementaria, la satisfacción percibida del desempeño realizado con el mapa propuesto obtuvo una mejor evaluación en el cuestionario de usabilidad PSSUQ, con una puntuación promedio de 2.0 en comparación con el mapa actual, cuyo resultado fue de 3.0; puesto que 1.0 es la mejor puntuación en relación con la usabilidad que se pudiera otorgar y 7.0 la peor, según la escala del cuestionario aplicado.

\section{Consideraciones finales}

Los resultados condujeron a presentar el mapa propuesto como la solución de diseño de información, debido a que cuenta con recomendaciones de la literatura que demostraron ser efectivas para aumentar la facilidad de uso del mapa entre los adultos mayores. Esta solución toma en cuenta todas las opiniones generadas por los participantes en las dos pruebas, haciendo de éste, un proceso de diseño participativo y centrado en el usuario, que ayuda a satisfacer la necesidad de conocimiento y despierta la curiosidad e interés por el contenido, de manera que se disminuya la confusión y la frustración entre los usuarios. Para que el diseño de información sea exitoso tendrá que generar un alto grado de satisfacción en el usuario [31], el diseño de información propuesto lo logra, dando pie a una etapa en la cual se pretende trasladar esta información (aplicada a todas las rutas) a una plataforma web o tipo APP para que se encuentre disponible a través de distintos dispositivos electrónicos, aumentando así la accesibilidad a la información tanto a adultos mayores, jóvenes y otros grupos poblacionales, satisfaciendo esa carencia de este tipo de información.

\section{Referencias}

[1] Group, N. N. Usability 101: Introduction to Usability. City, 2016.

[2] United Nations, D. o. E. a. S. A., Population Division. World Population Ageing 2013. United Nations, New York, 2013.

[3] Organization, W. H. Active ageing: a policy framework: a contribution of the World Health Organization to the Second United Nations World Assembly on Ageing. Madrid (ES): WHO (2002).

[4] Organization, W. H. Global age-friendly cities: A guide. World Health Organization, 2007.
[5] Seidel, D., Crilly, N., Matthews, F. E., Jagger, C., Brayne, C. and Clarkson, P. J. Patterns of functional loss among older people: a prospective analysis. Human Factors: The Journal of the Human Factors and Ergonomics Society (2009).

[6] Hounsell, N., Shrestha, B., Mcdonald, M. and Wong, A. Open data and the needs of older people for public transport information. Transportation Research Procedia, 14 (2016), 4334-4343.

[7] Nations, U., Affairs, D. o. E. a. S. and Development, D. f. S. P. a. Accessibility for the Disabled, A Design Manual for a Barrier Free Environment. City, 2003.

[8] Aceves-Gonzalez, C., Cook, S. and May, A. Inclusive service design: in search of better services. City, 2014.

[9] Grotenhuis, J.-W., Wiegmans, B. W. and Rietveld, P. The desired quality of integrated multimodal travel information in public transport: Customer needs for time and effort savings. Transport Policy, 14, 1 (2007), 27-38.

[10] Horn, R. E. Information design: Emergence of a new profession. Information design (1999), 15-33.

[11] Vu, K.-P. L. and Proctor, R. W. Handbook of human factors in Web design. CRC Press, 2011.

[12] Nielsen, J. Usability engineering. Elsevier, 1994.

[13] Nielsen, J. Enhancing the explanatory power of usability heuristics. ACM, City, 1994.

[14] Hörold, S., Mayas, C. and Krömker, H. User-oriented information systems in public transport. Contemporary Ergonomics and Human Factors (2013), 160-167.

[15] Horan, T. A., Abhichandani, T. and Rayalu, R. Assessing user satisfaction of e-government services: development and testing of quality-in-use satisfaction with advanced traveler information systems (ATIS). IEEE, City, 2006.

[16] Wall, G. and McDonald, M. Improving bus service quality and information in Winchester. Transport Policy, 14, 2 (2007), 165-179.

[17] ISO, W. 9241-11. Ergonomic requirements for office work with visual display terminals (VDTs). The international organization for standardization, 45 (1998).

[18] Waller, S., Bradley, M., Hosking, I. and Clarkson, P. J. Making the case for inclusive design. Applied ergonomics, 46 (2015), 297-303.

[19] Kawahara, K. and Narikawa, M. The unique achievements of Japanese industries in the super-aged society. Applied ergonomics, 46 (2015), 258-266.

[20] (INEGI), I. N. d. E. y. G. Censo de población y vivienda 2010. City, 2010.

[21] Barrett, J. Support and information needs of older and disabled older people in the UK. Applied Ergonomics, 36, 2 (2005), 177-183.

[22] Nielsen, J. and Loranger, H. Prioritizing web usability. Pearson Education, 2006.

[23] Krug, S. Don't make me think: A common sense approach to web usability. Pearson Education India, 2005.

[24] Ewing, R. and Bartholomew, K. Pedestrian \& TransitOriented Design, 2013. 
[25] Lynch, K. La imagen de la ciudad Barcelona. Editorial Gustavo Gili (1998).

[26] Hendrick, H. W. and Kleiner, B. M. Macroergonomics, Theory, Methods, and Applications. CRC PressTaylor \& Francis Group, 2005.

[27] Lewis, J. R. IBM computer usability satisfaction questionnaires: psychometric evaluation and instructions for use. International Journal of Human-Computer Interaction, 7, 1 (1995), 57-78.
[28] Persad, U., Langdon, P. and Clarkson, J. Characterising user capabilities to support inclusive design evaluation. Universal Access in the Information Society, 6, 2 (2007), 119-135.

[29] Robinson, P. A. Writing and Designing Manuals and Warnings 4e. CRC Press, 2009.

[30] Prado León, L. C., Rosalio Percepción Visual IIAplicaciones para el diseño. Editorial Universitaria, México, 2010.

[31] DeLone, W. H. and McLean, E. R. Information systems success revisited. IEEE, City, 2002 